\title{
Ensemble behaviour in population processes with applications to ecological systems
}

\author{
${ }^{1}$ Pollett, P.K. \\ ${ }^{1}$ Department of Mathematics, University of Queensland, Qld 4072, Australia. E-Mail: pkp@ maths.uq.edu.au
}

Keywords: Stochastic models, Markov chains, population processes

\begin{abstract}
We study Markovian models for population processes in continuous time, addressing questions concerning the behaviour of ensembles of individuals (equilibrium, quasi-equilibrium and time-dependent behaviour), and in particular what can be deduced from models for individual behaviour. It is self evident that ensemble behaviour is precisely the combined behaviour of individuals, so let me be more precise by way of three examples of populations, which will be used throughout to illustrate our major results. I will make the distinction between the two kinds of models (or processes) by referring to them simply as the "individual model" or the "ensemble model" (or process).
\end{abstract}

Population 1. Our first example is a population network, frequently called a metapopulation (see for example Gilpin and Hanski (1991)), where a fixed number $n$ of individuals occupies geographically separated regions or patches. Patches may become empty, but can be recolonized through migration from other patches. From the point of view of the individual, it spends a period of time in a given patch and might then emigrate to another patch, spend a period there, and so forth. Assuming individuals do not affect each other's progress through the network, one could model the progress of the individual as a random walk on the patches, and thus evaluate quantities such as the probability $p_{j}(t)$ that the individual occupies patch $j$ at time $t$. Our intuition tells us that, for the ensemble, the proportion of individuals in patch $j$ should be approximately equal to $p_{j}(t)$. So strong is this intuition that scientists frequently model population proportions using individual-level models.

In Section 2 we give a careful examination of whether it is reasonable to approximate random proportions of individuals that share a characteristic using probabilities derived from individual-based models. We are able to make a very precise statement for a very general class of models.

Population 2. This is a variant of Population 1 where we allow death or external emigration from any patch. We will study two cases: (i) the open network, where there is external immigration to one or more patches, and (ii) the closed network, where there are $n$ individuals to begin with, each eventually disappearing from the network through death or external emigration. As before, individuals are assumed not to affect one another's progress, but now individuals (perhaps arriving from outside the network) perform a random walk on the patches but then eventually leave. In contrast to Population 1, the total number of individuals is random. Yet, we would expect to be able to draw similar conclusions concerning ensemble proportions. Furthermore, as the population would be expected to settle down to a stable equilibrium, we might ask whether it is also reasonable to approximate the equilibrium proportion of individuals occupying patch $j$ using the equilibrium probability that an individual is in patch $j$. This is certainly reasonable in the open case, but even closed metapopulations can exhibit "quasi" equilibrium behaviour over reasonable time scales before extinction occurs; see Pollett (1999).

We examine these questions in Sections 3 and 4 evaluating stationary quasi-stationary distributions for the open and closed ensembles, respectively, and describing their relationship with the corresponding distributions for the individual model.

Population 3. Our final example is a population of organisms, each having a life time that consists of several distinct stages (for example, the butterfly life cycle comprises egg, larva, pupa and adult). Again our intuition tells us that the proportion of the population in stage $s$ should be close to the proportion of time $p_{s}$ that an individual spends in stage $s$ of its life cycle. Results proved in Section 2 confirm this. The quasiequilibrium behaviour of this population is examined in Section 4

We begin by describing a general individual model and then construct the corresponding ensemble model. The individual model is Markovian with a specified set of transition rates $Q$. Since $Q$ is arbitrary, the model is very flexible. In the ensemble model individuals are assigned to the various states, each then moving independently according to $Q$. 


\section{INDIVIDUAL AND ENSEMBLE MODELS}

Let $X(t)$ be the state of an individual at time $t(\geq 0)$; for Populations 1 and 2 it is the patch occupied by that individual, while for Population 3 it is the current stage in the individual's life cycle. We will suppose that $(X(t), t \geq 0)$ is a continuous-time Markov chain taking values in a discrete set $S$ with a (conservative) set of transition rates $Q=\left(q_{i j}, i, j \in S\right)$. Thus, $q_{i j}$ represents the rate of transition from state $i$ to state $j$, for $j \neq i$, and $q_{i}:=-q_{i i}=\sum_{j \neq i} q_{i j}$ represents the total rate out of state $i$. The sample behaviour is as follows: the process starts in a given state $i$, spends an exponentially distributed amount of time there with mean $1 / q_{i}$ before jumping to another state $j$ with probability $p_{i j}=q_{i j} / q_{i}$, where it spends another exponentially distributed period, but with mean $1 / q_{j}$, and so on. If the process reaches a state $j$ for which $q_{j}=0$ (an absorbing state) it remains there. For further explanations and terminology see for example Anderson (1991) or Norris (1997). It will be convenient to restrict our attention to the case where $S$ is a finite set, but I note that many of the arguments presented hold more generally. The state probabilities $\boldsymbol{p}(t)=\left(p_{j}(t), j \in S\right)$, where $p_{j}(t)=\operatorname{Pr}(X(t)=j)$, can be obtained as the (unique) solution to the forward equation $\boldsymbol{p}^{\prime}(t)=\boldsymbol{p}(t) Q$ satisfying $\boldsymbol{p}(0)=\boldsymbol{a}$, where $\boldsymbol{a}=\left(a_{j}, j \in S\right)$ is a given initial distribution (here and henceforth all vectors shall be interpreted as row vectors).

Population 1. Suppose that there are $M$ patches in the population network. The states $S=\{1, \ldots, M\}$ are the patch labels and $q_{i j}$ represents the intrinsic tendency for movement of individuals from patch $i$ to patch $j$ : when the individual leaves patch $i$ it moves to patch $j$ with probability $p_{i j}=q_{i j} / q_{i}$, where $q_{i}=\sum_{j \neq i} q_{i j}$ is the total (per-capita) rate of departure from patch $i$. For example, $q_{i j}$ might be a function of the area of patch $i$ and the distance between patches $i$ and $j$, but our results do not require any particular prescription. We will suppose only that $S$ is irreducible: any individual may pass between any two patches either directly or indirectly via a chain of other patches.

Population 2. Now $S=\{0, \ldots, M\}$ and we have the extra transition from $i$ to 0 at (per-capita) rate $q_{i 0}$ for each $i \in C:=\{1, \ldots, M\}$, corresponding to death or external emigration at patch $i$. We will suppose that $q_{i 0}>0$ for at least one $i$. In the open case there is an additional set of parameters $\boldsymbol{\nu}=$ $\left(\nu_{i}, i \in C\right)$ with $\nu_{i}$ representing the rate at which new individuals arrive at patch $i$ : when the individual starts its journey through the network it begins in patch $i$ with probability $a_{i}:=\nu_{i} / \sum_{k \in C} \nu_{k}$. In contrast to the model for Population 1, $S$ here is not irreducible because 0 is an absorbing state. However, it will be convenient to suppose that $C$ is irreducible: the individual can leave the network from any patch or, in the open case, reach any patch from outside the network (in both cases either directly or via a chain of other patches).

Population 3. In the life cycle example we let $M$ be the number of stages of life, with $M$ being the first stage and 1 being the last, and with state 0 representing death, so that $S=\{0, \ldots, M\}$. The rate at which an individual moves from stage $s$ to the next stage $s-1(s \geq 1)$ is $q_{s}=q_{s, s-1}>0$ (equivalently, the individual spends an exponentially distributed amount of time in stage $s$ with mean $1 / q_{s}$ ). Again $S$ is not irreducible. Each state $s$, including the absorbing state 0 , is in a class by itself.

Now we construct a model for the ensemble. Suppose that at time $t=0$ the individuals are assigned to the states according to some rule and then each moves independently in $S$ as a Markov chain governed by $Q$. The key assumption here is independence: individuals do not affect one another. We record only the number of individuals in the various states, rather than their positions. Let $N_{j}(t)$ be the number of individuals in state $j$ at time $t$, and let $\boldsymbol{N}=\left(N_{j}, j \in S\right)$. The process $(\boldsymbol{N}(t), t \geq 0)$ is also a continuous-time Markov chain. In applied probability parlance, $\boldsymbol{N}(t)$ is an example of a migration process (Whittle (1967)), or, in queueing theory parlance, a network of infiniteserver queues. Suffice it to say that systems like these are much studied and well understood. Books by Kelly (1979) and Serfozo (1999) together give an excellent summary of stochastic network theory. Since we are assuming that individuals move independently, the ensemble model can also be viewed as a (non-interacting) particle system, and thus dates back to at least Doob (1953).

The closed ensemble. We suppose that there is a fixed number $n$ of individuals, each moving according to $Q$. Population models 1 and 3 and the closed version of model 2 fit this context. The ensemble process takes values in $E=\left\{\boldsymbol{n} \in\{0, \ldots, n\}^{S}: \sum_{j \in S} n_{j}=n\right\}$, and its transition rates $Q_{E}=(q(\boldsymbol{n}, \boldsymbol{m}), \boldsymbol{n}, \boldsymbol{m} \in E)$ are given by $q\left(\boldsymbol{n}, \boldsymbol{n}+\boldsymbol{e}_{j}-\boldsymbol{e}_{i}\right)=n_{i} q_{i j}$, for all states $j \neq i$ in $S$, where $e_{j}=(0, \ldots, 0,1,0, \ldots, 0)$ is the unit vector with a 1 as its $j$-th entry. This follows because a transition from $\boldsymbol{n}$ to $\boldsymbol{n}+\boldsymbol{e}_{j}-\boldsymbol{e}_{i}$ is effected by the movement of a single individual from state $i$ to state $j$ and there are $n_{i}$ individuals in state $i$ when the state is $\boldsymbol{n}$, each moving to state $j$ at rate $q_{i j}$. Notice that the total rate out of state $\boldsymbol{n}$ is

$$
q(\boldsymbol{n}):=\sum_{\boldsymbol{m} \in E: \boldsymbol{m} \neq \boldsymbol{n}} q(\boldsymbol{n}, \boldsymbol{m})=\sum_{i \in S} n_{i} q_{i} \quad(\boldsymbol{n} \in E) .
$$

The open ensemble. Now individuals may enter the system and, once there, move according to $Q$. New individuals appear in the various states according to independent Poisson processes, the rate for state $j$ being $\nu_{j}$. With Population 2 (open case) in mind, we 
will adopt the setup $S=\{0\} \cup C$, where $C$ is a finite irreducible class and 0 is an absorbing state which is accessible from $C\left(q_{i 0}>0\right.$ for at least one $\left.i \in C\right)$, so that an individual's departure from the system is effected by its entry into state 0 . Thus, the ensemble process takes values in $E=\{0,1, \ldots\}^{C}$, which is irreducible because $C$ is irreducible, and we have additional sets of transition rates $q\left(\boldsymbol{n}, \boldsymbol{n}-\boldsymbol{e}_{i}\right)=n_{i} q_{i 0}$ and $q\left(\boldsymbol{n}, \boldsymbol{n}+\boldsymbol{e}_{i}\right)=\nu_{i}(i \in C)$. Now the total rate out of state $\boldsymbol{n}$ is

$$
\begin{aligned}
q(\boldsymbol{n})= & \sum_{i \in C} \nu_{i}+\sum_{i \in C} n_{i} \sum_{j \in C, j \neq i} q_{i j} \\
& +\sum_{i \in C} n_{i} q_{i 0}=\nu+\sum_{i \in C} n_{i} q_{i} \quad(\boldsymbol{n} \in E),
\end{aligned}
$$

where $q_{i}=\sum_{j \in S, j \neq i} q_{i j}(i \in C)$ and $\nu=\sum_{i \in C} \nu_{i}$.

\section{ENSEMBLE PROPORTIONS}

The closed ensemble. Our aim is to demonstrate that the proportion of individuals occupying state $j$ can indeed be approximated by the probability that a single individual is in state $j$. To this end let $X_{j}(t)=$ $N_{j}(t) / n$ be the proportion of individuals in state $j$ at time $t$, where recall that $n$ is the total number of individuals in the ensemble. Note that $(\boldsymbol{X}(t), t \geq 0)$, where $\boldsymbol{X}=\left(X_{j}, j \in S\right)$, is itself a continuoustime Markov chain. As we are going to vary $n$, let us make the dependence on $n$ explicit in our notation by writing $X_{j}^{(n)}$ for $X_{j}$ and $\boldsymbol{X}^{(n)}$ for $\boldsymbol{X}$, et cetera. Our first theorem establishes that, in the limit as $n$ becomes large, the process of proportions converges (uniformly in probability over finite time intervals) to a deterministic (that is, non-random) trajectory, being the unique solution of the forward equations (referred to above) of the individual model. The only proviso is that the initial proportions (at $t=0$ ) converge.

The proof of Theorem 1, along with the proofs of our other major results, are given in the appendix. Theorem 1, and Theorem 2 below, exploit important results of Kurtz (1970) on approximating limits of pure-jump Markov processes by solutions of ordinary differential equations.

Theorem 1. If $\boldsymbol{X}^{(n)}(0) \rightarrow \boldsymbol{a}$ as $n \rightarrow \infty$, then, for all $u>0$, and for every $\epsilon>0$,

$$
\operatorname{Pr}\left(\sup _{0 \leq t \leq u}\left|\boldsymbol{X}^{(n)}(t)-\boldsymbol{p}(t)\right|>\epsilon\right) \rightarrow 0 \text { as } n \rightarrow \infty,
$$

where $\boldsymbol{p}(t)=\left(p_{j}(t), j \in S\right)$ is the unique solution to $\boldsymbol{p}^{\prime}(t)=\boldsymbol{p}(t) Q$ satisfying $\boldsymbol{p}(0)=\boldsymbol{a}$, namely $\boldsymbol{p}(t)=$ $\boldsymbol{a} \exp (t Q)$, where $\exp (\cdot)$ is the matrix exponential.

The implications for our population models are obvious. For Model 1 and the closed version of Model 2, the proportion of individuals in patch $j$ at time $t$ can be approximated by the probability $p_{j}(t)$ that an individual occupies patch $j$ at time $t$, the individual moving amongst the patches according to any given irreducible (finite) Markov chain. For Population 3, the same is true but for the proportions of individuals in the various stages of life. But, as individuals follow a death process, we can exhibit the limiting proportions explicitly. For example, if $q_{1}, q_{2}, \ldots$ are distinct, then (tweak Exercise 6.8.31 of Grimmett and Stirzaker (2001)) the limiting proportions are given by

$$
\begin{array}{r}
p_{j}(t)=\frac{1}{q_{j}} \sum_{k=j}^{M} q_{k} e^{-q_{k} t} \prod_{l=j, l \neq k}^{M} \frac{q_{l}}{q_{l}-q_{k}}, \\
\text { for } j=1, \ldots, M \text {, and } p_{0}(t)=1-\sum_{j=1}^{M} p_{j}(t) .
\end{array}
$$

The open ensemble. Now the total number of individuals is not fixed but random, yet there is an analogue of Theorem 1, which provides an approximation that is valid for large external arrival rates (in the specific context of Population 2, heavy immigration from outside the population network). As we are going to let the total exogenous arrival rate $\nu$ become large, we make the dependence on $\nu$ explicit in our notation. Let $\boldsymbol{v}^{(\nu)}=\left(v_{j}^{(\nu)}, j \in\right.$ $C)$, where $v_{j}^{(\nu)}=\nu_{j} / \nu$ is the probability that, when a new individual appears, it appears in state $j$. Our premise is that $\boldsymbol{v}^{(\nu)} \rightarrow \boldsymbol{v}=\left(v_{j}, j \in C\right)$ as $\nu \rightarrow$ $\infty$. Similarly we let $\boldsymbol{X}^{(\nu)}=\left(X_{j}^{(\nu)}, j \in C\right)$, where $X_{j}^{(\nu)}(t)=N_{j}(t) / \nu$.

Theorem 2. Suppose that $\boldsymbol{v}^{(\nu)} \rightarrow \boldsymbol{v}$ as $\nu \rightarrow \infty$. Then, if $\boldsymbol{X}^{(\nu)}(0) \rightarrow \boldsymbol{a}$, we have, for all $u>0$ and, for every $\epsilon>0$,

$$
\operatorname{Pr}\left(\sup _{0 \leq t \leq u}\left|\boldsymbol{X}^{(\nu)}(t)-\boldsymbol{r}(t)\right|>\epsilon\right) \rightarrow 0 \text { as } \nu \rightarrow \infty
$$

where $\boldsymbol{r}(t)=\left(r_{j}(t), j \in C\right)$ is the unique solution to $\boldsymbol{r}^{\prime}(t)=\boldsymbol{v}+\boldsymbol{r}(t) Q_{C}$ satisfying $\boldsymbol{r}(0)=\boldsymbol{a}$, with $Q_{C}$ being the restriction of transition rate matrix $Q$ to $C$.

Notice that $\sum_{k \in C} X_{k}^{(\nu)}(t)=N(t) / \nu$, where $N(t)=\sum_{j \in C} N_{j}(t)$ is the total number of individuals in the system at time $t$. Therefore (formally), the proportion $N_{j}(t) / N(t)$ of individuals in state $j$ can be approximated by $r_{j}(t) / \sum_{k \in C} r_{k}(t)$. The quantity $r_{j}(t)$ is clearly not a probability, but does it admit a probabilistic interpretation for the individual model? It certainly does in the important special case where $\boldsymbol{a}=\mathbf{0}$, which happens if the ensemble system is initially empty. We can solve the differential equation in Theorem 2: since $\int_{0}^{t} \exp \left(u Q_{C}\right) d u Q_{C}=\exp \left(t Q_{C}\right)-I$, $\boldsymbol{r}(t)=\boldsymbol{a} \exp \left(t Q_{C}\right)+\int_{0}^{t} \boldsymbol{v} \exp \left(u Q_{C}\right) d u$. Thus $r_{j}(t)$ is the sum of two terms, the first being the probability that the individual is in state $j$ at time $t$ after his arrival under the initial distribution $\boldsymbol{a}$, and the second being 
the expected amount of time the individual spends in state $j$ up to time $t$ under the initial distribution $\boldsymbol{v}$ (the latter being the interpretation of $r_{j}(t)$ when $\boldsymbol{a}=\mathbf{0}$ ). This observation also permits us to make a connection with an important result of Kingman (1969). If the system is initially empty, then, remarkably, the numbers of individuals $N_{j}(t), j \in C$, in the various states are independent with $N_{j}(t)$ having a Poisson distribution with mean $\nu r_{j}(t)$; no approximation is required. It follows that $\mathbb{E}\left(X_{j}^{(\nu)}(t)\right)=r_{j}(t)$ for all $\nu$.

Before leaving this section we remark that Kurtz (1971) provides a Central Limit Theorem that allows one to approximate fluctuations of the process of proportions about its macroscopic limit. These fluctuations follow a Gaussian diffusion $\boldsymbol{Z}=(\boldsymbol{Z}(t), t \geq 0)$ whose parameters can be determined. For example, in the context of Theorem 1, the process of scaled proportions $\left(\boldsymbol{Z}^{(n)}(t), t \geq 0\right), \quad$ where $\boldsymbol{Z}^{(n)}(t)=\sqrt{n}\left(\boldsymbol{X}^{(n)}(t)-\boldsymbol{p}(t)\right)$ can be shown to converge (over finite time intervals) to $\boldsymbol{Z}$. This implies, for example, that $\left(\boldsymbol{X}^{(n)}\left(t_{1}\right), \ldots, \boldsymbol{X}^{(n)}\left(t_{k}\right)\right)$ has, for large $n$, an approximate Gaussian (normal) distribution with mean $\boldsymbol{p}(t)$ and a covariance structure that can be can be exhibited explicitly. Some of the implications of this for parameter estimation in population models are detailed in Ross et al. (2006).

\section{EQUILIBRIUM BEHAVIOUR}

The closed ensemble. When $S$ is irreducible (as it is in the model for Population 1), both the individual model and the ensemble model have a unique equilibrium distribution (because $S$ is finite). For the individual model the equilibrium distribution is the unique probability distribution $\boldsymbol{p}=$ $\left(p_{j}, j \in S\right)$ satisfying $\boldsymbol{p} Q=0 ; p_{j}$ is the equilibrium probability that the individual is in state $j$. For the ensemble model the equilibrium distribution $\boldsymbol{\pi}^{(n)}=$ $\left(\boldsymbol{\pi}^{(n)}(\boldsymbol{n}), \boldsymbol{n} \in E\right)$ is given by

$$
\boldsymbol{\pi}^{(n)}(\boldsymbol{n})=n ! \prod_{j \in S} \frac{p_{j}^{n_{j}}}{n_{j} !} \quad(\boldsymbol{n} \in E) .
$$

To see this, apply Theorem 2.3 of Kelly (1979). So, in equilibrium, the numbers of individuals $N_{j}, j \in S$, in the various states have a multinomial distribution with parameters $\left(p_{j}, j \in S\right)$; it is as if the $n$ individuals were allocated to the various states independently, with state $j$ being allocated with probability $p_{j}$.

Since the two equilibrium distributions are also limiting (they are the limit of the state probabilities as $t \rightarrow \infty$ ), we might expect the equilibrium proportions in the ensemble model to converge to $\boldsymbol{p}$ as $n \rightarrow \infty$. Whilst this cannot be deduced from Theorem 1, because uniform convergence in probability is established over finite time intervals, it is obviously true from elementary considerations. Indeed a stronger result obtains. Suppose that $\left(N_{j}, j \in S\right)$ has the multinomial distribution (1). Then, $N_{j}$ has a binomial distribution with parameters $n$ and $p_{j}$. Hence, by the Strong Law of Large Numbers, $N_{j} / n \rightarrow p_{j}$ with probability 1 . Indeed, $\boldsymbol{N} / n \rightarrow \boldsymbol{p}$ with probability 1 . So for large ensembles the equilibrium proportions of individuals in the various states can be approximated by proportions that accord with the equilibrium distribution of the individual model.

The open ensemble. In equilibrium, the numbers of individuals $N_{j}, j \in C$, in the various states are independent, with $N_{j}$ having a Poisson distribution with mean $\alpha_{j}:=\nu r_{j}$, where $\boldsymbol{r}=\left(r_{j}, j \in\right.$ $C)$ is the unique solution to $\boldsymbol{v}+\boldsymbol{r} Q_{C}=$ 0. This is a result due to Bartlett (1956) (and consistent with the result of Kingman mentioned earlier). Thus, the total number of individuals $N=\sum_{j \in C} N_{j}$ has a Poisson distribution with mean $\alpha:=\nu \sum_{j} r_{j}$ and, given $N=n,\left(N_{j}, j \in\right.$ $C)$ has the multinomial distribution (1), but with $p_{j}=r_{j} / \sum_{k \in C} r_{k} \quad\left(=\alpha_{j} / \alpha\right)$. It follows, again from elementary considerations, that the equilibrium proportions converge, as $\nu$ becomes large, to $\boldsymbol{p}$ (with probability 1$)$, where $\boldsymbol{p}=\left(p_{j}, j \in C\right)$.

\section{QUASI-EQUILIBRIUM BEHAVIOUR}

We restrict our attention to the following case: $S=\{0\} \cup C$, where $C$ is an irreducible finite set, and 0 is an absorbing state which is accessible from $C$ (we have in mind here the closed version of Population model 2). Each of $n$ individuals reaches 0 with probability 1 in finite mean time, and so too the ensemble process reaches its absorbing state $(n, 0, \ldots, 0)$ in finite mean time. However, the ensemble process is not irreducible. It has irreducible classes $\quad E_{k}=\left\{\boldsymbol{n} \in\{0,1, \ldots\}^{S}: \sum_{j \in C} n_{j}=k\right\}$ ( $k=0,1, \ldots, n)$ corresponding to there being $k$ individuals in $C$, with $E_{0}$ having the single member $(n, 0, \ldots, 0)$, the process moving from $E_{k}$ to $E_{k-1}$ when one of the $k$ individuals that remain in $C$ reaches 0 . The classes are therefore arranged as follows: $E_{n} \succ E_{n-1} \succ \cdots \succ E_{1} \succ E_{0}(D \succ E$ means $q_{i j}>0$ for at least one $i$ in $D$ and at least one $j$ in $E$ ).

Theorem 1 tells us that at any time $t$ the ensemble proportions can be approximated by $\boldsymbol{p}(t)=\boldsymbol{a} \exp (t Q)$, the entries of $\boldsymbol{a}$ being the (large- $n$ ) initial proportions of individuals in the various states. But, what happens when $t$ is large? Although both processes have degenerate equilibrium distributions assigning all mass to the absorbing state, they can settle down to a quasi equilibrium before extinction occurs. For this reason we usually model the long-term behaviour 
using a limiting conditional (or quasi-stationary) distribution. The idea is as follows. First suppose we have complete information: we know the probability $p_{x}(t)$ that the process is in state $x$ at time $t$, for all states $x$ and for every time $t$. If at a fixed time $t$ we observe that the process has not reached the absorbing state $z$, the appropriate probability to evaluate is $p_{x}(t) /\left(1-p_{z}(t)\right)$, the probability that the process is in state $x$ conditional on it not having been absorbed by time $t$; to be emphatic, this is the best possible information we could have about the chance of being in state $x$ having observed that the process has not been absorbed. This being the case for every time $t$, it is natural to evaluate this probability in the limit as $t \rightarrow \infty$ to obtain information about the long-term behaviour of the process conditional on non-absorption (hence the term limiting conditional distribution). Under mild conditions this limiting conditional distribution (LCD) can be determined, directly from the transition matrix restricted to the transient states, as an appropriately normalized left eigenvector corresponding to the eigenvalue with maximum real part; it is not necessary for us to know $p_{x}(t)$ explicitly.

If the transient states form an irreducible class $C$, as is the case in the individual model, we can apply the classical result of Darroch and Seneta (1967). The restricted transition matrix $Q_{C}$ has an eigenvalue $-\alpha$ with maximum real part, which is real, strictly negative and simple (multiplicity 1), and corresponding to it are strictly positive left and right eigenvectors, $\boldsymbol{u}=\left(u_{j}, j \in C\right)$ and $\boldsymbol{x}^{\top}=\left(x_{j}, j \in\right.$ $C)$. Furthermore, $p_{j}(t) /\left(1-p_{0}(t)\right) \rightarrow \pi_{j}$, where $\pi_{j}=u_{j} / \sum_{k \in C} u_{k}(j \in C)$. The quantity $\alpha$ is called the decay parameter, because $p_{j}(t)=O\left(e^{-\alpha t}\right)$ (see Kingman (1963)). For the (reducible) ensemble model, we must appeal to recent results of Van Doorn and Pollett (2007) to establish the existence of a LCD over the set of non-absorbing states $C_{E}:=$ $E \backslash E_{0}=\cup_{k=1}^{n} E_{k}$. Indeed, the ensemble process is an example of a quasi-death process, and Theorem 7 of Van Doorn and Pollett (2007) can be applied. The main result of this section shows that the LCD of the ensemble process assigns positive probability only to those states in $E_{1}$, being precisely the "unit vectors" $\left(n-1, e_{j}\right)=(n-1,0, \ldots, 0,1,0, \ldots, 0)(j \in$ $C)$ corresponding to the single remaining individual being in state $j$ (and hence $n-1$ in state 0 ).

Theorem 3. The ensemble process admits a LCD $\boldsymbol{u}_{E}=\left(u(\boldsymbol{m}), \boldsymbol{m} \in C_{E}\right)$. It assigns all its mass to $E_{1}$, with $u\left(\left(n-1, e_{j}\right)\right)=\pi_{j}(j \in C)$, where $\left(\pi_{j}, j \in C\right)$ is the LCD of the individual process.

Whilst this result is not surprising (because each of the $n$ individuals is lost at the same rate), we see that again ensemble proportions can be approximated by probabilities obtained from the individual model. It accords with Theorem 1; since the proportion $X_{j}^{(n)}(t)$ of individuals in state $j$ at time $t$ is approximately $p_{j}(t)$, we should expect $N_{j}(t) /\left(n-N_{0}(t)\right)=$ $X_{j}^{(n)}(t) /\left(1-X_{0}^{(n)}(t)\right)$, the proportion in state $j$ of those "alive" at time $t$, to be close to $p_{j}(t) /\left(1-p_{0}(t)\right)$ $(j \in C)$.

Van Doorn and Pollett (2007) contains enough technology to extend Theorem 3 to the case where $C$ is not irreducible, but where the (sole) absorbing state 0 is accessible from all states in $C$ for which the initial distribution assigns positive probability (however, note that now the ensemble process is not a quasi-death process). We will content ourselves with a result for Population 3 (which of course has $C$ reducible). Recall that there are $M$ stages of life, with $M$ being the first stage and 1 being the last, and with state 0 representing death. Individuals move from stage $s$ to stage $s-1$ at rate $q_{s}>0$. Theorem 6 of Van Doorn and Pollett (2007) gives the LCD of the individual process. Let $C=\{1, \ldots, M\}$ and $q=\min _{s \in C} q_{s}$. One might have expected that those stages $s$ for which $q_{s}=q$ ( $s$ could in fact be unique) would act as a "bottleneck" in that most individuals would be expected to be in those stages. But, it turns out that the limiting probability of being in stage $s$, conditional on being alive, is 0 unless $s \leq \kappa$, where $\kappa=\min \left\{k: q_{k}=q\right\}$.

Theorem 4. For the death process describing Population 3, the ensemble process admits a LCD $\boldsymbol{u}_{E}=\left(u(\boldsymbol{m}), \boldsymbol{m} \in C_{E}\right)$, which does not depend on the initial distribution over states. It assigns all its mass to $E_{1}$, with $u\left(\left(n-1, \boldsymbol{e}_{s}\right)\right)=\pi_{s}(s \in C)$, where $\left(\pi_{s}, s \in C\right)$, the LCD of the individual process, is given by

$$
\pi_{s}= \begin{cases}\frac{q}{q_{s}} \prod_{i=1}^{s-1}\left(1-\frac{q}{q_{i}}\right) & \text { if } s \leq \kappa \\ 0 & \text { if } s>\kappa .\end{cases}
$$

\section{SURVIVAL}

We conclude proceedings with a result that brings together many of the ideas of the paper.

One of the many characterizations of the LCD is that if a Markov process starts in state $x \in \mathcal{X}$ (some irreducible set) with probability $\pi_{x}$, where $\boldsymbol{\pi}=\left(\pi_{x}, x \in \mathcal{X}\right)$ is the LCD (conditional on not having left $\mathcal{X}$ ), then the chance that the process is in state $y \in \mathcal{X}$ at time $t$ is $\pi_{y} e^{-\alpha t}$, where $\alpha$ is the decay parameter of $\mathcal{X}$. This result holds in great generality, and, depending on the setting, usually results from a simple calculation; for a recent discussion, see for example Steinsaltz and Evans (2004). It follows immediately that the time to exit from $\mathcal{X}$ is exponentially distributed with parameter $\alpha$ (because the probability of still being in $\mathcal{X}$ at time $t$ is $\sum_{y \in \mathcal{X}} \pi_{y} e^{-\alpha t}=e^{-\alpha t}$ ). The 
implications for our models are obvious, the result for the ensemble being not particularly surprising because the ensemble survives until the last individual reaches 0 . However, using an elaboration of the arguments presented in Section 3 of Derman (1955) (see also the proof of Theorem 2 of Brown (1970) for a more transparent discrete-time parallel), we can prove the following. The setup is $S=\{0\} \cup C$, where $C$ is an irreducible finite set with decay parameter $\alpha$ and 0 is an absorbing state which is accessible from $C$.

Theorem 5. Let $\boldsymbol{\pi}=\left(\pi_{j}, j \in C\right)$ be the LCD of the individual process. If the initial numbers $N_{j}(0)$, $j \in C$, are chosen independently with $N_{j}(0)$ having a Poisson distribution with mean $\pi_{j}$, then, for all $t>0$, $N_{j}(t), j \in C$, are independent with $N_{j}(t)$ having a Poisson distribution with mean $\pi_{j} e^{-\alpha t}$.

For the aficionados. This result holds in much greater generality; $C$ need not be finite, $Q$ could be explosive, $\boldsymbol{\pi}=\left(\pi_{j}, j \in C\right)$ could be any $\alpha$-subinvariant measure and, more remarkably still, $\pi$ need not be finite (we could have $\sum_{j \in C} \pi_{j}=\infty$ ); for definitions, see Anderson (1991).

\section{APPENDIX}

Proof of Theorem 1. The ensemble model is density dependent in the sense of Kurtz (1970): there is an open subset of $\mathbb{R}^{S}$, namely $E=(0,1)^{S}$, and functions $f: E \times \mathbb{Z}^{S} \rightarrow \mathbb{R}$ with the property that $q(\boldsymbol{n}, \boldsymbol{n}+\boldsymbol{l})=n f(\boldsymbol{n} / n, \boldsymbol{l}), \boldsymbol{n}, \boldsymbol{n}+\boldsymbol{l} \in \mathbb{Z}^{S}$. Clearly $f\left(\boldsymbol{x}, \boldsymbol{e}_{j}-\boldsymbol{e}_{i}\right)=x_{i} q_{i j}, j \neq i$. Our theorem is then proved by applying Theorem 3.1 of Kurtz's paper. We first define $F: E \rightarrow \mathbb{R}^{S}$ by $F(\boldsymbol{x})=\sum_{\boldsymbol{l}} \boldsymbol{l} f(\boldsymbol{x}, \boldsymbol{l})$. This function is required to be Lipschitz continuous on $E$ (clearly true because $F$ has bounded first partial derivatives on $E$ ), thus guaranteeing a solution $\boldsymbol{p}(t)$ to $\boldsymbol{p}^{\prime}(t)=F(\boldsymbol{p}(t)), \boldsymbol{p}(t) \in E, 0 \leq t \leq u$, satisfying $\boldsymbol{p}(0)=\boldsymbol{a}$. Next, two technical conditions must be fulfilled: (i) $\sup _{\boldsymbol{x} \in E} \sum_{\boldsymbol{l} \neq \mathbf{0}}|\boldsymbol{l}| f(\boldsymbol{x}, \boldsymbol{l})<\infty$ and (ii) $\lim _{d \rightarrow \infty} \sup _{\boldsymbol{x} \in E} \sum_{|\boldsymbol{l}|>d}|\boldsymbol{l}| f(\boldsymbol{x}, \boldsymbol{l})=0$. They are trivially satisfied because $S$ is a finite set. Kurtz's theorem states that $\boldsymbol{X}^{(n)}(t)$ converges uniformly in probability over finite time intervals to $\boldsymbol{p}(t)$ as $n \rightarrow$ $\infty$, so it remains for us to show that $F(\boldsymbol{x})=\boldsymbol{x} Q$. This is straightforward:

$$
\begin{aligned}
F(\boldsymbol{x}) & =\sum_{i \in S} \sum_{j \neq i}\left(\boldsymbol{e}_{j}-\boldsymbol{e}_{i}\right) f\left(\boldsymbol{x}, \boldsymbol{e}_{j}-\boldsymbol{e}_{i}\right) \\
& =\sum_{k \in S} x_{k} \sum_{i \in S} q_{k i} \boldsymbol{e}_{i} .
\end{aligned}
$$

Hence, $F(\boldsymbol{x})=\boldsymbol{x} Q$, because (elementwise) $F_{i}(\boldsymbol{x})=$ $\sum_{k \in S} x_{k} q_{k i}(i \in S)$.

Proof of Theorem 2. In addition to $f\left(\boldsymbol{x}, \boldsymbol{e}_{j}-\boldsymbol{e}_{i}\right)=$ $x_{i} q_{i j}, j \neq i$, we have $f\left(\boldsymbol{x},-\boldsymbol{e}_{i}\right)=x_{i} q_{i 0}$ and $f\left(\boldsymbol{x}, \boldsymbol{e}_{j}\right)=v_{j}$ (asymptotically). The model is not strictly density dependent, but asymptotically density dependent in the sense of Pollett (1990), and the present result follows from Theorem 3.1 of Pollett's paper, which shows that $\boldsymbol{X}^{(\nu)}(t)$ converges uniformly in probability over finite time intervals to $\boldsymbol{r}(t)$ as $\nu \rightarrow$ $\infty$, the unique solution to $\boldsymbol{r}^{\prime}(t)=F(\boldsymbol{r}(t)), \boldsymbol{r}(t) \in E$, $0 \leq t \leq u$. We may evaluate $F(\boldsymbol{x})$ as follows:

$$
\begin{aligned}
F(\boldsymbol{x})= & \sum_{i \in C} \sum_{j \in C, j \neq i}\left(\boldsymbol{e}_{j}-\boldsymbol{e}_{i}\right) f\left(\boldsymbol{x}, \boldsymbol{e}_{j}-\boldsymbol{e}_{i}\right) \\
& -\sum_{i \in C} \boldsymbol{e}_{i} f\left(\boldsymbol{x},-\boldsymbol{e}_{i}\right)+\sum_{j \in C} \boldsymbol{e}_{j} f\left(\boldsymbol{x}, \boldsymbol{e}_{j}\right) \\
= & \sum_{k \in C} x_{k} \sum_{i \in C} q_{k i} \boldsymbol{e}_{i}+\sum_{i \in C} \boldsymbol{e}_{i} v_{i},
\end{aligned}
$$

where recall that $q_{i}=-q_{i i}=\sum_{j \in S, j \neq i} q_{i j}$. Hence, $F(\boldsymbol{x})=\boldsymbol{v}+\boldsymbol{x} Q_{C}$, because (elementwise) $F_{i}(\boldsymbol{x})=$ $v_{i}+\sum_{k \in C} x_{k} q_{k i}(i \in C)$.

Proof of Theorem 3. Let $Q_{k}$ be the restriction to $E_{k}$ of transition rate matrix $Q_{E}$ of the ensemble process and let $-\alpha_{k}$ be the eigenvalue of $Q_{k}$ with maximum real part $(k=1, \ldots, n)$. Then, $\alpha_{k}=k \alpha$. To see this, observe that $\alpha_{k}=\lim _{t \rightarrow \infty}-(1 / t) \log \operatorname{Pr}(T>$ $t$ ), where $T$ is the time to first exit of the process from $E_{k}$ (see Kingman (1963)); the limit does not depend on the initial distribution over states. However, $T=\min \left\{T_{1}, \ldots, T_{k}\right\}$, where $T_{i}$ is the time it takes individual $i$ to reach 0 , and, since the individuals move independently, $\operatorname{Pr}(T>t)=$ $\prod_{i=1}^{k} \operatorname{Pr}\left(T_{i}>t\right)$. Since the individuals move according to $Q,-(1 / t) \log \operatorname{Pr}\left(T_{i}>t\right) \rightarrow \alpha$ as $t \rightarrow \infty$. Hence, $\alpha_{k}=k \alpha$. It follows immediately that $-\alpha$ is the eigenvalue of $Q_{E}$ with maximum real part, and, moreover, that its algebraic, and hence geometric, multiplicity is equal to 1 . We may therefore appeal directly to Theorem 5 of Van Doorn and Pollett (2007), which implies that the LCD of the ensemble process exists provided the initial distribution assigns mass to at least one of $E_{1}, \ldots, E_{n}$ (we assume that all $n$ individuals are present initially, and so all this mass is assigned to $E_{n}$ ). Furthermore, the LCD is the unique non-negative solution $\boldsymbol{u}_{E}=$ $\left(u(\boldsymbol{m}), \boldsymbol{m} \in C_{E}\right)$ to $\boldsymbol{u}_{E} Q_{E}=-\alpha \boldsymbol{u}_{E}$ with $\boldsymbol{u}_{E} \mathbf{1}^{\top}=1$. So, it remains to show that the given $\boldsymbol{u}_{E}$ satisfies these equations: $u(\boldsymbol{m})=0$ unless $\boldsymbol{m} \in E_{1}$, in which case $u\left(\left(n-1, \boldsymbol{e}_{j}\right)\right)=\pi_{j}(j \in C)$, where $\boldsymbol{\pi}=\left(\pi_{j}, j \in C\right)$ is the LCD of the individual process, that is, the unique (strictly positive) solution to $\boldsymbol{\pi} Q=-\alpha \boldsymbol{\pi}$ with $\boldsymbol{\pi} \mathbf{1}^{\top}=1$. Writing out the latter we get $\sum_{i \in C, i \neq j} \pi_{i} q_{i j}=\left(q_{j}-\alpha\right) \pi_{j}(j \in C)$, where $q_{j}=\sum_{k \in S, k \neq j} q_{j k}$, while the former eigenvector equation may be written as

$$
\sum_{\boldsymbol{m} \in E_{n}, \boldsymbol{m} \neq \boldsymbol{n}} u(\boldsymbol{m}) q(\boldsymbol{m}, \boldsymbol{n})=(q(\boldsymbol{n})-\alpha) u(\boldsymbol{n}),
$$

$\boldsymbol{n} \in E_{n}$, and, for $k=1, \ldots, n-1$,

$$
\begin{gathered}
\sum_{\boldsymbol{m} \in E_{k+1}} u(\boldsymbol{m}) q(\boldsymbol{m}, \boldsymbol{n})+\sum_{\boldsymbol{m} \in E_{k}, \boldsymbol{m} \neq \boldsymbol{n}} u(\boldsymbol{m}) q(\boldsymbol{m}, \boldsymbol{n}) \\
=(q(\boldsymbol{n})-\alpha) u(\boldsymbol{n}) \quad\left(\boldsymbol{n} \in E_{k}\right) .
\end{gathered}
$$


Clearly (2), and (3) for $k=2, \ldots, n$, are satisfied because $u(\boldsymbol{m})=0$ when $\boldsymbol{m} \in \cup_{k=2}^{n} E_{k}$, and the remaining equation $(k=1)$ will hold if and only if

$$
\begin{aligned}
& \sum_{j \in C} \sum_{i \in C, i \neq j} u\left(\boldsymbol{n}+\boldsymbol{e}_{j}-\boldsymbol{e}_{i}\right)\left(n_{j}+1\right) q_{j i} \\
&=\left(\sum_{k \in C} n_{k} q_{k}-\alpha\right) u(\boldsymbol{n}) \quad\left(\boldsymbol{n} \in E_{1}\right),
\end{aligned}
$$

remembering that the total rate out of state $n \in E_{k}$ is $q(\boldsymbol{n})=\sum_{i \in C} n_{i} q_{i}$. But, $\boldsymbol{m} \in E_{1}$ if and only if $\boldsymbol{m}=\left(n-1, \boldsymbol{e}_{i}\right)$ for some $i \in C$, and so we require

$\sum_{i \in C, i \neq j} u\left(\left(n-1, \boldsymbol{e}_{i}\right)\right) q_{i j}=\left(q_{j}-\alpha\right) u\left(\left(n-1, \boldsymbol{e}_{j}\right)\right)$,

for $j \in C$. On substituting $u\left(\left(n-1, \boldsymbol{e}_{i}\right)\right)=\pi_{i}$, we find that $\sum_{i \in C, i \neq j} \pi_{j} q_{i j}=\left(q_{j}-\alpha\right) \pi_{j}(j \in C)$, which of course is the individual process eigenvector equation.

Proof of Theorem 4. This is similar to the proof of Theorem 3, but now we appeal to Theorem 3 of Van Doorn and Pollett (2007) for important information about accessibility; the initial distribution must assign all mass to $\cup_{k \geq \kappa_{E}} E_{k}$, where $\kappa_{E}$ is the smallest index $k$ of those $\alpha_{k}$ which share the smallest value among $\alpha_{1}, \ldots, \alpha_{n}$. However, $\alpha_{k}=k \alpha$, implying that $\kappa_{E}=1$, and so the accessibility condition is satisfied automatically (we have assumed that the ensemble process starts in $E_{n}$ ). Evaluating the LCD as directed by Theorem 3 of Van Doorn and Pollett (2007) amounts to calculating $\boldsymbol{u}_{E}$ as in the proof of our Theorem 3, but now the individual process is a simple death-process. Therefore, $\alpha=q$ and the unique (strictly positive) solution $\pi=\left(\pi_{j}, j \in C\right)$, to $\boldsymbol{\pi} Q=-\alpha \boldsymbol{\pi}$ with $\boldsymbol{\pi} \mathbf{1}^{\top}=1$ is given in Theorem 6 of Van Doorn and Pollett (2007), and written out in the statement of our theorem.

\section{REFERENCES}

Anderson, W.J. (1991), Continuous-Time Markov Chains: An Applications-Oriented Approach, Springer-Verlag, New York.

Bartlett, M.S. (1956), Deterministic and stochastic models for recurrent epidemics, In Proceedings of the 3rd Berkeley Symposium on Mathematical Statistics and Probability, 1954-1955, Vol. IV, Pages 81-109, Berkeley and Los Angeles, University of California Press.

Brown, M. (1970), A property of Poisson processes and its application to macroscopic equilibrium of particle systems, The Annals of Mathematical Statistics, 41, 1935-1941.

Darroch J.N. and E. Seneta (1967), On quasistationary distributions in absorbing continuoustime finite Markov chains, Journal of Applied Probability, 4, 192-196.
Derman, C. (1955), Some contributions to the theory of denumerable Markov chains, Transactions of the American Mathematical Society, 79, 541-555.

Doob, J.L. (1953), Stochastic Processes, John Wiley \& Sons Inc., New York.

Gilpin, M.E. and I. Hanski (1991), Metapopulation Dynamics, Academic Press, New York.

Grimmett, G.R. and D.R. Stirzaker (2001), Probability and Random Processes, 3rd edition, Oxford University Press, New York.

Kelly, F.P. (1979), Reversibility and Stochastic Networks, Wiley, Chichester.

Kingman, J.F.C. (1963), The exponential decay of Markov transition probabilities, Proceedings of the London Mathematical Society, 13, 337-358.

Kingman, J.F.C. (1969), Markov population processes, Journal of Applied Probability, 6, 1-18.

Kurtz, T.G. (1970), Solutions of ordinary differential equations as limits of pure jump Markov processes, Journal of Applied Probability, 7, 49-58.

Kurtz, T.G. (1971), Limit theorems for sequences of jump Markov processes approximating ordinary differential processes, Journal of Applied Probability, 8, 344-356.

Norris, J.R. (1997), Markov Chains, Cambridge University Press, Cambridge.

Pollett, P.K. (1990), On a model for interference between searching insect parasites., Journal of the Australian Mathematic Society, Series B, 31, 133 150.

Pollett, P.K. (1999), Modelling quasi-stationary behaviour in metapopulations, Mathematics and Computers in Simulation, 48, 393-405.

Ross, J.V., Taimre, T. and P.K. Pollett (2006), On parameter estimation in population models, Theoretical Population Biology, 70, 498-510.

Serfozo, R. (1999), Introduction to Stochastic Networks, Springer-Verlag, New York.

Steinsaltz, D. and S.N. Evans (2004), Markov mortality models: implications of quasistationarity and varying initial distributions, Theoretical Population Biology, 65, 319-337.

Van Doorn, E.A. and P.K. Pollett (2007), Survival in a quasi-death process, Technical Report, The University of Queensland: http://www.maths.uq.edu.au/ pkp/papers/survival/ survival.pdf

Whittle, P. (1967), Nonlinear migration processes, Bulletin of the International Institute of Statistics, 42, 642-647.

\section{ACKNOWLEDGEMENT}

The work of Phil Pollett is supported by the Australian Research Council Centre of Excellence for Mathematics and Statistics of Complex Systems. 